\title{
Le retour des migrants ou l'émergence des « nouveaux Ouzbeks » : les effets d'un rite de transition
}

Return of the Migrants or Apparition of the "New Uzbeks": The Effects of a Transition Ritual

El retorno de migrantes o la emergencia de los "nuevos Uzbecos": los efectos de un rito de transición

\section{Sophie Massot}

\section{(2) OpenEdition}

\section{Journals}

Édition électronique

URL : https://journals.openedition.org/remi/5208

DOI : 10.4000/remi.5208

ISSN : $1777-5418$

Éditeur

Université de Poitiers

Édition imprimée

Date de publication : 1 décembre 2010

Pagination : 59-84

ISBN : 978-2-911627-56-9

ISSN : 0765-0752

Référence électronique

Sophie Massot, "Le retour des migrants ou l'émergence des « nouveaux Ouzbeks » : les effets d'un rite de transition », Revue européenne des migrations internationales [En ligne], vol. 26 - n³ | 2010, mis en ligne le 01 décembre 2013, consulté le 15 avril 2022. URL : http://journals.openedition.org/remi/ 5208 ; DOI : https://doi.org/10.4000/remi.5208 


\title{
Le retour des migrants ou l'émergence des «nouveaux Ouzbeks »: les effets d'un rite de transition
}

\author{
Sophie MASSOT ${ }^{1}$
}

\section{INTRODUCTION}

T es migrations de travail des Ouzbeks ${ }^{2}$ vers Séoul, Moscou et New York ont _fait l'objet de recherches ${ }^{3}$ menées de 2007 à 2010 (Massot, 2009). Nous avons montré que les migrants aux différentes étapes du parcours migratoire se trouvent au centre d'une nébuleuse socio-familiale dont les intérêts économiques et les enjeux de pouvoir sont complexes et n'agissent pas toujours avec la même prégnance. Il convient

1 Docteur en Science Politique, ATER de Science Politique à l'Université de la Réunion, 11 bis rue Guy Saint Lambert, 97411 Bois de Nèfles-Saint Paul ; sophiealdric@yahoo.fr

2 Il convient ici de distinguer les termes « ouzbek » (qui renvoie à la nationalité) et « ouzbékistanais » (qui renvoie à la citoyenneté). J'ai choisi de me concentrer sur l'émigration des Ouzbeks d'Ouzbékistan, et non sur l'ensemble des Ouzbékistanais. Chaque nationalité a sa spécificité, ce qui induit des processus migratoires difficilement comparables. Chacun a un lien particulier avec l'Ouzbékistan, qui peut représenter un pays d'origine, d'accueil, d'exil, de destination volontaire ou forcée. Il est ardu de mêler dans une même analyse la mobilité des Ouzbékistanais de nationalité allemande, ouzbèke ou coréenne. Par ailleurs, les Ouzbékistanais ne sont pas tous perçus de la même façon à l'étranger. Ainsi, un Ouzbékistanais russe et un Ouzbékistanais ouzbek ne rencontreront pas les mêmes difficultés à Moscou. Enfin, bien que cela soit sujet à débat, il semble que chaque groupe ethnique cultive sa propre culture, qui peut avoir une influence considérable sur la manière d'envisager la migration, puis sur la période migratoire. Une autre raison à ce choix de m'intéresser à la nationalité ouzbèke est qu'elle est majoritaire en Ouzbékistan, bien que les chiffres officiels soient à manier avec précaution. L'impact des migrations des Ouzbeks sur leur pays d'origine est peut-être plus sensible, surtout dans un contexte où une politique d'ouzbékisation a été menée.

3 Dans le cadre du doctorat à l'IEP de Paris, plusieurs terrains ont été abordés : recherche préparatoire en Ouzbékistan (juillet-août 2005) qui a permis de mesurer les enjeux des migrations internationales, puis Moscou (avril-mai 2006), Séoul (juillet-août 2006), New York (février-mars 2007) et enfin Ouzbékistan (avril-mai 2007 et janvier-février 2009). Retour en Ouzbékistan en janvier-février 2009 afin de mesurer l'impact des retours des migrants. 
alors de considérer ces diverses relations nouées par les migrants selon un cadre spatiotemporel qui permet d'en mesurer l'évolution : il faut distinguer la période liminaire en Ouzbékistan, qui précède le départ, de la période où les migrants se trouvent dans le pays d'accueil, et enfin lorsqu'ils reviennent à leur point de départ ; la plupart des migrations étant temporaires. De même, il faut distinguer les liens de nature familiale et ceux de nature socio-économique.

Il est possible d'analyser ce phénomène à l'aune de ce que j'ai appelé un « rite de transition », notion forgée à partir des travaux de Van Gennep (1981) sur les rites de passages et de Bourdieu (1982) sur les rites d'institution. En effet, la migration effective représente un rite qui doit s'accomplir seul après la mise en place par le réseau familial des conditions de sa réalisation. Dès lors, lorsque les migrants séjournent dans le pays d'accueil, ils doivent se prouver à eux-mêmes et à leur famille qu'ils appartiennent bien à leur communauté afin de refonder leur identité ouzbèke - leur ouzbékité/o'zbekchilik (Massot, 2009). La rupture avec les réseaux d'origine serait donc une condition nécessaire à la validation de la migration par le groupe d'origine, et à la réintégration du migrant dans ce réseau, qui implique une prise de pouvoir socio-économique.

Nous nous intéresserons ici au retour des migrants, en nous demandant quels sont les effets de ce rite de transition lorsque la boucle migratoire a été réalisée : les migrants changent-ils de statut social en fonction de leur nouveau pouvoir économique ? Y a-t-il création de nouveaux réseaux de solidarité dont ils seraient le pivot? Leur propre perception de l'ouzbékité est-elle transformée par le parcours migratoire ? S'inscrivent-ils dans de nouvelles logiques migratoires?

Nous montrerons tout d'abord que les effets sociaux du rite de transition migratoire induisent un bouleversement des statuts au sein de la parenté, en nous demandant s'il y a importation de modèles culturels et familiaux occidentaux, puis nous analyserons l'émergence de la catégorie socio-économique des "nouveaux ouzbeks », ce qui nous permettra de souligner les paradoxes en matière d'investissement de l'argent rapporté par la migration. Il s'agit finalement de montrer que les migrants, au centre du rite de transition dont ils sont les acteurs, se désengagent peu à peu lors du retour et se replient sur ce qu'ils considèrent être leur ouzbékité.

\section{BOULEVERSEMENT ET RÉAMÉNAGEMENT DES STATUTS AU SEIN DE LA PARENTÉ : LES EFFETS SOCIAUX DU RITE DE TRANSITION}

Le départ et surtout l'absence du migrant ont des conséquences directes sur la famille restée au pays d'origine. On assiste à une paradoxale occidentalisation des proches qui, depuis l'Ouzbékistan, cherchent à travers les médias occidentaux de nouveaux modèles de vie. Les valeurs et fonctions familiales changent, la place du père, du frère ou de l'époux restant inexploitée en raison de la migration. De retour, le migrant devra retrouver une nouvelle place dans l'organisation domestique. 


\section{Vers une occidentalisation de la famille?}

La première conséquence visible de l'émigration est le faible pourcentage d'hommes dans certains lieux ${ }^{4}$. Samarkand est actuellement réputée pour être majoritairement habitée de femmes, d'enfants et de personnes âgées. Il s'agit sans aucun doute d'une exagération, mais cette impression souligne néanmoins une réalité tangible : de très nombreux hommes sont partis à l'étranger et leur absence pousse ceux qui restent à réorganiser le quotidien en fonction des places laissées vides. Il s'agit à la fois des postes laissés vacants ${ }^{5}$, mais aussi des places sociales : l'éloignement du père, du mari, du fils ou du frère n'est pas sans conséquence sur l'organisation domestique, sur le statut et les fonctions des différents membres de la parenté voire sur la définition même de la famille.

\section{Vers une parentalisation des grands-parents}

Dans bien des cas, les épouses des migrants s'installent avec leurs enfants chez leurs beaux-parents. Les parents des migrants accueillent leur bru et leurs petits-enfants dans la maison familiale et exercent l'autorité sur l'ensemble des habitants du logement. Cet état de fait s'explique par l'usage en Ouzbékistan de la résidence virilocale. Après le mariage, les époux gagnent généralement le domicile des parents du marié, avant de s'installer dans un logement indépendant. Seul le plus jeune fils reste traditionnellement chez ses parents jusqu'à leur décès. Mais dans le cas des migrations, c'est à nouveau chez ses beaux-parents que se retrouve bien souvent l'épouse du migrant. Elle y est soumise à l'autorité tutélaire de son beau-père, mais surtout à celle de sa belle-mère qui régente les activités domestiques. L'épouse se retrouve donc seule avec ses beaux-parents et ne peut compter sur son époux pour pacifier les relations. Elle n'a que peu de pouvoir décisionnel, hormis peut-être sur ses enfants, bien que ce ne soit pas toujours le cas, les grands-parents ayant leur mot à dire quant à l'éducation de leurs petits-enfants. Ils sont parfois amenés à prendre celle-ci totalement en charge. Il arrive en effet que des couples gagnent ensemble l'étranger, laissant leurs enfants chez leurs grands-parents en Ouzbékistan. C'est le cas de Farangiz, à Boukhara, qui héberge ses trois petits-enfants :

"Mon fils cadet est parti à Séoul il y a maintenant quatre ans. Au début, il était tout seul là-bas, mais il a voulu que ma bru vienne le rejoindre. Elle est partie là-bas depuis six mois. Leurs trois enfants sont restés ici avec nous, car c'est compliqué de partir avec eux. Et puis, il faut qu'ils grandissent ici ».

4 « Expert on labour migration from the Centre for Socioeconomic Research Lyudmila Maksakova believes that from 500000 to 800000 people of Uzbekistan's 27.6 million people are currently working abroad. However, this figure is not final, because in many Uzbek regions, especially in rural areas, adult members in almost every family leave for neighbouring countries to do seasonal jobs every year, leaving the elderly, women and children at home. At least one adult from other families works in foreign countries. ", http://www.uznews.net/news_single.php?lng=en\&cid=8\&nid=307, consulté le 17/07/2008.

5 Quatre migrants ouzbeks en Russie racontent au journaliste une des conséquences de l'émigration en Ouzbékistan : «À Geshvodan, notre village dans le sud de l'Ouzbékistan, il ne reste plus un seul homme. Tous partis en Russie! Cela pose un problème pour enterrer les morts, car la religion musulmane impose que ce soient les hommes qui posent les corps dans les tombes. La situation est devenue grotesque », http://www.bastamag.org/journal/article.php3?id_article=175, consulté le 17/07/2008. 
En effet, les migrants économiques n'emmènent pas leurs enfants à l'étranger, l'investissement que représenterait leur départ ne pouvant s'avérer lucratif alors qu'ils ne sont pas en âge de travailler. De plus, les migrants qui font l'objet de mes recherches n'ont pas vocation à s'installer durablement dans le pays d'accueil, et laissent donc en Ouzbékistan leur famille. Les grands-parents sont non seulement amenés à héberger leurs petits-enfants, parfois à les éduquer et s'en occuper au quotidien, mais également, pour certains, à les assumer financièrement. Certains migrants, et particulièrement ceux qui sont partis pour la Russie, ne donnent parfois aucune nouvelle pendant des années. Ce phénomène est intéressant, car il remet en question la place des parents comme éducateurs et perturbe la notion de foyer, où deux générations cohabitent, en l'absence de celle des parents. Ce bouleversement va de pair avec un changement dans le comportement des enfants.

\section{Changement de statut des enfants}

Les enfants en Ouzbékistan se trouvent en position inférieure par rapport aux aînés. Selon Poujol : "la première question posée lors d'une rencontre entre deux individus qui ne se connaissent pas concerne leur année de naissance respective afin de déterminer qui a l'ascendant sur l'autre » (2006 : 11). Le respect se traduit entre autres par un vouvoiement dû aux personnes plus âgées, même si la différence d'âge est infime. On peut aussi lire dans les termes d'adresses cette insistance sur l'âge. Si un frère aîné est appelé $a k a$, un frère plus jeune est désigné par $u k a$. Pour une sœur plus âgée, il convient de dire opa, mais singil pour une sœur plus jeune. Ce respect des plus âgés présente cependant une particularité : une jeune mariée se doit de vouvoyer les enfants de la famille de son mari nés avant son mariage, et d'être tutoyée par eux. La nouvelle arrivée dans la famille a donc un statut inférieur au plus jeune des enfants de la parenté. Hormis cette exception, le respect des enfants envers les aînés, qu'ils soient ou non de leur famille, est considéré comme une clef de voûte de la culture ouzbèke. Le respect et l'obéissance au sein de la famille apparaissent comme une évidence pour mes interlocuteurs. Cependant, ils semblent quelque peu mis à mal par l'éloignement du migrant. En effet, certains notent une nouvelle attitude des enfants " sans père », qui seraient amenés à remettre en cause les principes d'éducation de leurs parents. C'est ce que déplore Gulnoza :

"Entre quatorze et dix-huit ans, certains jeunes sont bizarres. C'est vrai, ce $n$ 'est pas facile, leurs parents les voient comme des enfants alors qu'ils se sentent déjà adultes. Mais le problème, c'est quand ils sentent un déséquilibre dans la famille, alors là, c'est trop mauvais. Ils s'occupent en traînant dans la rue et en allant dans les vidéos-bars avec leurs copains. Parfois même, ils consomment de la drogue. Mais c'est parce qu'ils n'ont rien à faire pendant leurs vacances ! Avant, les jeunes allaient travailler avec leurs pères, comme ça ils ne faisaient pas n’importe quoi et en plus ils apprenaient un vrai métier. Mais maintenant, tout le monde est parti à l'étranger et leurs mères pleurent d'être toutes seules et les vieux se plaignent de devoir tout faire à la maison... Et puis, les gamins, tu leur dis d'apprendre un métier pour devenir quelqu'un de bien, un vrai homme sérieux, mais qu'est-ce que tu peux leur dire quand ils te disent qu'il n'y a pas besoin de faire des études pour partir à Moscou, et qu'ils préfèrent aller travailler quelque temps ailleurs plutôt que de devenir professeur ici et de ne pas avoir de quoi nourrir ta famille, même acheter du pain?». 
Ainsi, un souffle de rébellion verrait le jour dans la nouvelle génération d'adolescents ouzbeks, qui refuse le carcan traditionnel familial au profit d'une vie « à l'occidentale », selon leur propre dire. Manger des hamburgers ou des hot-dogs, aller en discothèque, jouer aux jeux vidéos en ligne dans les cybercafés, fumer, regarder des films érotiques seraient autant d'actes signant le rejet de la culture ouzbèke, du respect de l'autorité familiale et l'attrait pour un monde occidental fantasmé, dont les États-Unis seraient la métonymie, et la Russie la fenêtre d'accès vers cet univers. Poujol livre cette analyse : " quant à la crise d'adolescence officiellement inexistante dans ces pays de forte soumission aux aînés, il n'est pas dit que son coût social ne rejaillisse pas sur les générations plus âgées, sous la forme passive de la toxicomanie ou de l'alcoolisme et sous celle violente, du choix de l'extrémisme politico-religieux» (2006 : 11). Il semble que la crise d'adolescence, qui n'était pas une réalité culturelle auparavant en Ouzbékistan, fasse son apparition dans certaines familles de migrants. L'autorité paternelle est mise à mal par l'absence du père. C'est ce que raconte Šahnoza à propos de son cousin, qui s'est avéré être son frère biologique :

«Mon oncle et ma tante [maternels] ont donné à ma mère une fille, car elle $n$ 'arrivait pas à avoir d'enfant. Mais après, ma mère a réussi à avoir des enfants, et elle a donné à sa sœur [la tante maternelle de Šahnoza] un de ses fils, car ma tante n'arrivait pas à avoir de garçon. Ça arrive souvent ici, et on n'en parle pas vraiment, mais ça ne pose pas de problème, normalement. Mais mon oncle est parti pendant cinq ans en Russie, sans donner de nouvelles. Et mon cousin a appris à ce moment-là qu'il n'était pas son fils, enfin ... pas son fils de sang, quoi. Quand mon oncle est revenu, on était tous très heureux de le voir vivant. Mais mon cousin n'a pas accepté tout ça, et a commencé à lui répondre, à le regarder dans les yeux.

$C$ 'est vraiment la honte pour mon oncle, c'est vraiment difficile. Bon, là, il y a cette histoire qu'il n'a pas très bien prise. Mais on voit de plus en plus de jeunes qui $n$ 'obéissent plus trop bien à leurs parents, parce qu'ils ne les ont pas beaucoup vus à la maison, et ne sont plus habitués à leur obéir ».

Émerge ainsi une nouvelle catégorie, celle des adolescents, qui est marquée par un certain refus des habitudes ouzbèkes et une réticence face à l'autorité des parents, et en particulier du migrant qui, quand il revient, fait figure d'étranger auprès de ses enfants.

\section{Nouvelle place des femmes}

Le Comité pour l'élimination de la discrimination à l'égard des femmes des Nations Unies a montré du doigt l'Ouzbékistan, où « le poids des traditions et de l'idéologie patriarcale [constitue un] frein à l'émancipation des femmes $»^{6}$. Répondant aux experts, qui soulignaient les différences professionnelles entre hommes et femmes en Ouzbékistan, la délégation ouzbékistanaise a rétorqué que «dans les pays orientaux, une telle opposition entre la place des femmes dans la société, et celle des femmes dans la famille n'a pas lieu d'être. La femme a une fonction extrêmement sociale, qui est d'élever

6 Comité pour l'élimination de la discrimination à l'égard des femmes, « Le poids des traditions et de l'idéologie patriarcale en Ouzbékistan, frein à l'émancipation des femmes, inquiète fortement les experts », 10/08/2006, http://www.un.org/News/fr-press/docs/2006/FEM1574.doc.htm, consulté le 17/07/2008. 
ses enfants. Elle n'est pas prise en otage par sa famille, mais elle est mère, et membre actif de la société. Il s'agit avant tout d'un mode de vie ${ }^{7}$. Cet échange officiel apporte un éclairage sur la place de la femme en Ouzbékistan : la principale demande sociale qui lui est adressée est de mettre au monde des enfants, de les élever, de s'occuper des tâches domestiques et de ne pas porter atteinte à l'honneur de sa famille. Cela reste le cas aujourd'hui, néanmoins, un des changements majeurs dans l'organisation familiale est sans doute la nouvelle place occupée par certaines femmes dans la société. Cela se traduit par des actes qu'elles réalisent désormais, en l'absence des hommes, mari ou père, partis à l'étranger. C'est ce que résume Imrona :

"Maintenant, à Samarkand, on peut voir beaucoup de femmes dans les cafés et les restaurants. Des tablées de femmes, sans homme! Elles boivent même de la vodka, et tout! De toute façon, il ne reste plus que des femmes et des enfants, ici.

Tous les hommes sont partis gagner de l'argent ailleurs. Alors elles s'occupent. Elles ne peuvent pas attendre que les hommes reviennent pour vivre, c'est normal qu'elles sortent. Mais avant, cela ne se passait pas comme ça. C'est les hommes seulement qui sortaient aux restaurants. Parfois ils emmènent leur épouse, mais elle ne sort pas dans des lieux comme ça toute seule, ce n'est pas comme en France! ».

Les femmes des migrants auraient donc fait évoluer les mœurs féminines, vers un déplacement de la sphère domestique à la sphère publique. Cette dernière, généralement dirigée par les hommes, est investie par des femmes qui ne peuvent plus compter sur leurs époux, absents, pour organiser des sorties dans les lieux publics. De la même façon, certaines femmes de migrants décident de conduire une voiture personnelle.

"Avec l'argent envoyé par Ališer, on a acheté une voiture, une Nexia [de la marque Daewoo]. Mon fils l'utilise parfois, mais il n'est pas là souvent. Alors j'ai décidé de me mettre à conduire. J'ai pris quelques cours et ça va. Enfin..., j'ai peur quand même! [rires]. Mais c'est mieux pour moi, autrement je n'irais plus dans notre datcha. Et puis les transports en commun où on est serré, ce n'est pas pour moi. Les taxis, oui, mais autant avoir sa voiture particulière " [Nodira].

Passer son permis de conduire, décider de l'orientation du budget familial, exercer un métier prenant ou encore aller dans un café sont autant d'actions, considérées a priori comme réservées aux hommes, que des femmes de migrants font pourtant. En l'absence de l'autorité masculine, elles sont amenées à prendre des décisions qui reviendraient ordinairement à leur époux.

\section{Remise en question de l'autorité masculine}

Les migrants qui posent le pied sur la terre d'origine éprouvent des sentiments contradictoires : au soulagement et au plaisir de retrouver tout ce qui a manqué pendant l'exil se mêle une sensation de dépossession de ce qui les entoure. La place des hommes, puisque ce sont eux qui émigrent en majorité, a été transformée pendant la migration.

7 Ibid.

REMI 2010 (26) 3 pp. 59-84 


\section{L'étonnement du migrant devant les changements}

Les migrants qui reviennent sont d'abord surpris des changements au sein de leur famille, et des nouveautés architecturales de leur lieu d'origine. Beaucoup disent leur étonnement lors des retrouvailles avec leurs enfants grandis ou d'autres parents et ces changements leur font prendre conscience du temps passé en migration. Les travaux effectués dans la ville ou le village d'origine les surprennent aussi. Enfin, certains notent avec stupeur la raréfaction des hommes en Ouzbékistan, " tous partis à l'étranger », comme ils le disent eux-mêmes. Pour Ziëd, alors qu'il me présente une des enseignantes de physique-chimie de l'université de Namangan :

"Quand je suis parti, elle était mon étudiante. Maintenant, je suis revenu et elle est ma collègue! [rires]. C'est idiot, mais ça m'a fait très bizarre à la rentrée de discuter des cours avec elle. Je me suis dit "que de chemin parcouru!" Et j'étais en Corée pendant tout ce temps-là. C'est la vie! [en français]».

Le « chemin parcouru », c'est à la fois celui du migrant à Séoul et celui, parallèle, de son ancienne étudiante à Namangan. Mais ce qui marque particulièrement les migrants, qui l'expriment à demi-mot ou par le biais de l'humour, c'est le sentiment d'une perte de valeurs dans la famille d'origine, et d'un déclassement de leur autorité. Ils le craignaient parfois déjà depuis l'étranger, comme Ališer, rencontré à New York :

"Je ne comprends pas très bien l'évolution des choses, des fois. Je vois bien que mes enfants sont très attachés au fait d'avoir des marques de vêtements à la mode.

Ils n'en ont jamais assez! Je ne sais pas combien il faut avoir de pantalons pour être à la mode, mais il faudra bientôt acheter une maison pour pouvoir ranger leurs affaires, je pense! [rires]. À chaque fois que je téléphone, j'apprends qu'ils sont allés faire les magasins. Mais moi, dans mon village, je n'avais qu'un seul change, et je ne me posais pas de questions sur ça. Aujourd'hui, pour les jeunes, c'est très différent. Ma femme m'a dit que ma fille, il n'est pas question qu'elle porte une robe ouzbèke [ici, renvoie aux robes fabriquées sur mesure à partir d'un coupon de tissu]. Il faut absolument qu'elle aille acheter un vêtement chinois, ou turc, ou je ne sais pas quoi. Mais quand même, c'est nos traditions, il faut garder ça ».

Ce besoin des enfants d'obtenir des produits dont ne disposaient pas leurs parents dans leur enfance revient souvent dans le discours des migrants, qu'ils soient encore à l'étranger ou déjà revenus. Vêtements à la mode, mais aussi appareils électroniques sont devenus l'apanage des enfants des migrants, qui cherchent à se démarquer des générations précédentes en s'occidentalisant, en se rapprochant des cultures russes et américaines, par les habits ou la musique.

Poujol note l'apparition "de comportements "d'enfants gâtés" dans les milieux urbains favorisés du Kazakhstan, s'apparentant à "l'enfant-roi” de la société chinoise en plein développement et que l'on peut rapprocher du contexte ouzbek. Ainsi, autant l'amélioration des conditions de vie pour une fraction réduite de la population ajoutée à l'ouverture brutale des médias sur le monde extérieur (accès à Internet, parabole, diffusion des films étrangers) ont pu avoir des conséquences psychologiques décelables sous forme de pathologies connues en Occident (addiction aux jeux vidéo, crises de délire), la préca- 
risation brutale d'une part croissante de la population a nécessairement une traduction psychiatrique » (2006: 12).

Dans le cas des migrations, il y a bien la découverte par le migrant d'une crise familiale qui s'est produite à son insu, et qui entraîne une exigence d'un apport financier régulier, les dépenses générées par la famille étant désormais supérieures à celles précédant le départ.

\section{Changement du statut de père, de fils et d'époux}

Les migrants reviennent donc dans une famille où les enfants ont grandi, où l'épouse a pris une nouvelle place dans la sphère publique et où les parents assurent un rôle domestique nouveau. Ces changements de mœurs se sont opérés progressivement, mais sans que les migrants ne les perçoivent nécessairement depuis l'étranger. Il se retrouve donc face à une situation qui les isole. Cela engendre un décalage dans le rapport du migrant à sa famille :

" J'étais vraiment pressé de rentrer en Ouzbékistan. J'y pensais tout le temps à Séoul, je m'imaginais comment ce serait de se retrouver chez soi. Mais finalement,

ce n'est pas aussi simple que je le pensais. Bien sûr, je suis content d'être là, ce n'est pas ça. Mais les choses ont changé un peu pour moi. Je n'accepte plus comme avant que mes parents gèrent ma vie, me disent de faire ci ou ça avec mon argent. Et puis je pense qu'eux aussi ne me disent rien, mais ils trouvent que j'ai changé. Ils m'appellent parfois "le Coréen", pour rire. Je ne sais pas ce qui se passe exactement, mais je n'ai plus envie de travailler pareil que là-bas, et en même temps, ça me manque un peu. Car là-bas, je ne me posais pas autant de questions, je faisais ce que j'avais à faire, c'est tout » [Ilhom].

Plusieurs éléments sont à retenir dans ce récit et tout d'abord le sentiment de désœuvrement consécutif au retour. Les migrants ne retrouvent pas nécessairement une activité professionnelle à leur arrivée en Ouzbékistan, et beaucoup s'accordent une période de repos. Le rythme de travail jugé éreintant dans le pays d'accueil est évoqué avec regret par certains migrants qui se sentent désormais inutiles et sans objectifs. En migration il s'agissait de travailler pour amasser de l'argent et revenir au pays, une fois ce stade atteint les incitations à travailler sont beaucoup moins fortes. Ilhom note aussi son changement d'attitude face à ses parents. Il n'accepte plus les remarques qui lui sont adressées et le manque de liberté qu'il éprouve est nouveau. L'éloignement lui a fait oublier l'autorité parentale omniprésente et omnipotente, qu'il rechigne désormais à respecter. Pour Poujol : «La fin du système soviétique a produit une rupture éthique et comportementale due à la perte brutale des repères dans des sociétés auparavant fortement structurées. Cette rupture ne s'est pas accompagnée d'explications ni de dialogues entre les générations, parents-enfants notamment. Le non-dit a été la règle, à part quelques commentaires au sein des classes d'âge qui ont un grand rôle de structuration sociale en Asie centrale. À ceci ont succédé assez rapidement des considérations nostalgiques de la part des générations de retraités qui constatent de façon récurrente : "avant, cela ne se passait pas comme ça...” $"(2006: 12)$. 
Ce changement de comportement consécutif à la migration est même craint par certains parents, qui rechignent à laisser partir leur enfant à l'étranger de peur que cette expérience ne le fasse évoluer négativement. Ainsi pour Nodira :

"Tous mes enfants parlent anglais, et ma deuxième fille a eu l'occasion de partir aux États-Unis. Mais je n'ai pas voulu. Elle m'en veut à cause de ça. Mais d'abord, je ne voulais pas qu'elle prenne l'avion, c'est trop dangereux. Et puis quand même, je connais ma fille, mais on ne sait jamais ce qu'il peut arriver là-bas, elle peut changer de comportement. Je veux que mes enfants continuent à être bien élevés, à baisser la tête et à obéir à leurs parents, même s'ils sont adultes. Et à ne pas regarder leur père dans les yeux. Ceux qui partent, on ne les reconnaît plus quand ils rentrent, ils sont devenus effrontés, sans respect. »

À ce generation gap, pour reprendre le terme anglo-saxon, entre le migrant et ses propres parents, s'ajoute celui entre le migrant et ses propres enfants, qui eux-mêmes ne s'inclinent plus devant l'autorité du migrant. Dès lors, quelle place peut occuper le migrant au sein de sa famille?

\section{Se taire et s'individualiser}

Le migrant s'est bien souvent construit une image de l'Ouzbékistan basée essentiellement sur une nostalgie de la patrie quittée et de l'entourage familial regretté. Il a également élaboré un investissement symbolique important quant à une tradition ouzbèke, que l'on a qualifié d'ouzbékité, qui reposerait sur des valeurs morales opposées à la «nonculture » des pays d'accueil. Or inversement, les membres de sa famille ont souhaité profiter des capitaux étrangers pour s'occidentaliser à la manière ouzbèke, s'il est possible de le décrire ainsi. Les femmes sont devenues plus indépendantes en investissant la sphère publique, en choisissant des biens de consommation visibles par tous, en opérant des actes jugés virils, tels que celui de conduire ou de s'arrêter à une terrasse de café. Les enfants des migrants se sont tournés vers ce qu'ils estiment être une culture américaine ou européenne, en choisissant des vêtements, de la nourriture et des attitudes qu'ils jugent à la mode.

Tout le paradoxe de la migration est là : l'argent amassé en migration sert, en Ouzbékistan, à imiter un style de vie étranger, que n'a pourtant pas adopté le migrant dans le pays d'accueil, puisque celui-ci s'est au contraire tourné vers la culture du pays d'origine. Les migrants n'évoquent que très peu leur expérience migratoire. Peu de précisions sont apportées sur la façon dont ils ont vécu la migration. À ce sujet on peut se référer à Temine (1999: 136) qui mentionne les « dix commandements destinés [...] à faciliter l'insertion des travailleurs immigrés dans la société française », parus en janvier 1899 dans le journal Emigrato. Le dixième commandement dit : «de retour au pays, ne dis pas de mal de la terre qui t'a nourri, ni des hommes qui ont vécu avec toi ».

De la même façon, suivant ce précepte tacite, les migrants n'évoquent presque jamais leur travail en migration. Les familles ne posent d'ailleurs pas de questions sur les conditions matérielles dans le pays d'accueil. Le migrant constate donc à la fois combien son absence a conduit à des remaniements familiaux importants, combien la période 
migratoire a été vécue différemment par ses proches et par lui-même et, parallèlement, il n'évoque qu'à demi-mot cette constatation souvent mêlée de dépit. Pour retrouver une nouvelle place dans la société, il lui faut mettre en avant son enrichissement, afin que son statut d'homme « qui vit bien » soit admis par tous, sans pour autant dévoiler les moyens dont il a usé pour parvenir à cette fin. Il est devenu " nouvel Ouzbek », et une nouvelle charge sociale lui incombe désormais : celle de faire fructifier ses réseaux nouvellement acquis.

\section{Émergence des « nouveaux Ouzbeks » : la ligne de séparation créée par le rite d'institution}

À Séoul, après avoir fait étalage de ses deux téléphones portables, de ses mails qu'il dit consulter tous les jours, du restaurant ouzbek de Paris qu'il a découvert sur Internet, de son amour pour les beaux vêtements et de son rêve de voir un jour la Tour Eiffel, Odil me demande d'estimer son âge et, juste après, veut savoir s'il ressemble à un Ouzbek... Que répondre et que veut-il entendre ? Qu'il ne ressemble pas à un Ouzbek car la migration l'a changé en occidental?

Comme le note Centlivres (2000: 5), dans les sociétés à cheval entre plusieurs États, "le processus identitaire déborde également le choix entre deux identités nationales. Bref, diasporas, migrations et collectivités "transmigrantes" sont des figures d'une nouvelle normalité $d u$ fonctionnement des sociétés modernes ». On peut alors se demander comment cela se traduit pour les migrants ouzbeks de retour dans le giron familial.

\section{Historique de cette catégorie officieuse}

Au cours des entretiens réalisés, tant dans les pays d'accueil que dans le pays d'origine, j'ai pu constater qu'il n'existait pas de terme général pour désigner les migrants. J'utilisais pour ma part, en russe, celui de migranty, qui était compris par mes interlocuteurs, mais rarement repris à leur compte. Les migrants ne se désignent pas en tant que tel, ne se représentent pas comme groupe collectif car ils ne veulent pas exister en tant que « migrants ». Ils se définissent davantage comme Ouzbeks quand ils sont dans le pays d'accueil. Mais comment désigner ceux qui sont rentrés?

En réalité, la migration apparaît comme une phase d'entre-deux, où les migrants sont en transition vers un nouvel état : ils ne relèvent déjà plus vraiment de leur ancien statut qui serait celui d'Ouzbeks en recherche de mieux-vivre, et pas encore intégrés dans celui qu'il recherche, c'est-à-dire celui d'Ouzbeks enrichis revenus dans leur pays d'origine. Comment appeler ces anciens migrants rentrés en Ouzbékistan avec un changement visible de mode de vie et de consommation ? Un terme semble progressivement s'imposer : celui de «nouvel Ouzbek ». Il est sans aucun doute un dérivé de l'expression russe novyj russkij [r. : litt., nouveau Russe], qui avait lui-même repris l'expression russe novorič dérivée du français « nouveau riche ». En russe, ces deux expressions renvoient aux entrepreneurs et aux oligarques qui se sont brusquement enrichis à la chute de l'URSS, souvent par le biais d'actions illégales et en partenariat avec des réseaux mafieux. 
En Ouzbékistan, l'expression « nouvel Ouzbek » évoque surtout l'idée d'un parvenu, quelqu'un ayant subitement changé de statut financier et qui entend le faire savoir autour de lui. Cette expression est employée pour tous les cas d'enrichissement rapide. Elle ne relève pas nécessairement de l'ordre du compliment, bien que beaucoup envient cette position. Absence de discernement dans les dépenses et faste déployé dans le seul but d'impressionner l'entourage sont souvent critiqués par les témoins de ce changement. C'est ce que note avec ironie Nilufar :

«Notre voisin, je l'ai connu tout petit. Il jouait souvent avec mes enfants. Il $m$ 'appelait tata, on était proche. Il aimait bien rester manger à la maison, il était simple. Mais depuis qu'il est revenu de Corée, il se prend pour l'émir [rires] ! Il ne peut pas sortir sans son téléphone portable et, quand il arrive devant chez ses parents, il klaxonne soi-disant pour qu'on lui ouvre le portail, mais moi je crois plutôt que c'est pour montrer à tout le monde sa nouvelle Nexia».

Le changement financier est donc noté, mais accompagné d'une critique sévère du changement d'attitude. Comme l'explique un article de presse, l'Ouzbékistan est « une immense lessiveuse des profits illicites de toute la région. Seule une très petite partie de l'argent blanchi est investie sur place, mais il alimente des importations de produits de luxe : parfumerie française, vêtements de luxe et supermarchés d'alimentation hors de portée de l'immense majorité de la population. La force de ce dispositif, outre qu'il favorise l'émergence d'une classe relativement aisée, embauchée dans la nébuleuse des entreprises mafieuses, c'est qu'il s'enracine profondément dans la structure sociale du pays $\gg^{8}$. Les nouveaux Ouzbeks en sont les acteurs. Ainsi, le gendre d'Ulugbek et de Maftuna, à Tashkent, est-il prêt à dépenser l'équivalent de plusieurs salaires moyens en Ouzbékistan pour s'acheter des vêtements de manufacture européenne. Il s'agit d'avoir de l'argent et, surtout, de le faire savoir. Pour cela, il convient de se montrer à la fois généreux et de placer ses investissements dans des activités visibles.

\section{Un classement par l'argent}

Un classement social semble établi par l'argent, en Ouzbékistan, sans que soient mis en question les moyens d'acquisition de cette soudaine fortune. Comme le rappelle Amandine Regamey, qui analyse les histoires drôles, « le nouveau russe est un "nouveau riche” caractérisé par sa bêtise, son mauvais goût, mais ses énormes possibilités financières (acquises illégalement) qui lui permettent de tout acheter, femmes et policiers compris » (Regamey, 1999 : 5). De la même façon, un nouvel Ouzbek est caractérisé par son ascension financière fulgurante, mais dont l'origine est inconnue. Dans le cadre de la migration économique, il ne s'agit pas nécessairement de capitaux acquis via des réseaux mafieux, mais souvent illégalement, les migrants ayant pour beaucoup séjourné sans autorisation dans le pays d'accueil et n'ayant pas été déclarés par leurs employeurs. Par ailleurs, comme ils évoquent de manière très lacunaire leurs conditions de vie à l'étranger, celle-ci apparaît floue et auréolée de mystère.

8 http://www.voltairenet.org/article7396.html, consulté le 20/07/2008. 
C'est ce que soulignent aussi Bennegadi et Bourdillon (1990 : 131) à propos des migrants maghrébins en France : «on note souvent quelques difficultés pour le travailleur migrant qui revient [...] dans son groupe d'origine, à expliciter clairement le travail qu'il fait. Lorsque l'écart est trop grand symboliquement entre le statut social de la personne dans son groupe d'origine et celui dans le pays d'accueil, se développe toute une stratégie de non-dit où la complicité est totale, admise et opérante; on ne parle que du côté gratifiant de ce travail sans avoir à dire en quoi il consiste ».

L'important pour le nouvel Ouzbek est d'avoir accumulé une somme d'argent importante lui permettant de montrer à qui veut le voir son nouveau statut social. Cette ascension est étonnamment dépouillée de valeurs morales. Ainsi, si des habitants suspectent parfois certaines voisines d'avoir gagné l'Arabie saoudite pour s'y prostituer, et revenir dans leur pays d'origine avec des moyens nouveaux, ils considéreront néanmoins cet argent comme une marque de changement de statut. L'échelle de valeur n'est pas basée sur les valeurs morales, mais uniquement financières.

Il ne s'agit pas nécessairement d'une remarque positive. Pétric (2006 : 213-214) évoque le cas des entrepreneurs enrichis à la faveur de la perestroïka et explique qu' « il s'agit en général d'individus n'ayant pas fait d'études. [...] Ces individus, ayant fait parfois fortune très rapidement, bénéficient d'une grande autorité sociale grâce à leur carrière. [...] Les plus riches d'entre eux sont appelés yangi Uzbeklar ou novi Uzbeki (nouveaux Ouzbeks), en référence à l'expression "nouveau riche”, du fait de leur ascension sociale très rapide et de leur niveau culturel médiocre ». Être nouvel Ouzbek, c'est éprouver le besoin de montrer son argent, et cet étalage provoque à la fois des regards envieux, mais aussi des moqueries. C'est ce que m'explique Nodir qui évoque le cas de son oncle :

"Mon oncle, c'est vraiment mon modèle. Il est parti avec très peu d'argent, au départ. Mais il a fait du business, et comme c'est un très bon businessman, il a commencé à très bien gagner sa vie. Je voudrais être comme lui. Enfin, non, pas tout à fait, parce qu'il est vraiment devenu trop riche, et moi, je ne veux pas être un nouvel Ouzbek [rires]. Je ne veux pas que l'argent me monte à la tête, et sortir en cravate, les mains derrière le dos [rires]. Je suis trop jeune pour ça. Mais gagner de l'argent, oui. Et avoir de l'autorité [Il le dit en se tapant sur le ventre], car pour l'instant, ce n'est pas encore ça [rires] ».

Avoir de l'argent, c'est pouvoir se permettre d'adopter certaines attitudes, telles que « porter la cravate » ou « avoir les mains dans le dos », position des hommes respectables qui marchent d'un air assuré, ou encore « avoir de l'autorité », qui se matérialise par un ventre proéminent, signe que l'on a les moyens de beaucoup manger.

\section{Changement de réseaux sociaux et nouvelle vision politique}

Mais qu'apporte exactement ce changement de situation financière ? Que cache cette opulence exhibée à grand renfort d'ostentation? Sans doute la motivation première des migrants est-elle l'amélioration des conditions matérielles de leur famille. Mais comme le soulignent Adelkhah et Olszewska (2006) à propos des Afghans, la mobilité peut être envisagée « comme une stratégie sociale ». Avoir de l'argent, ce n'est pas unique- 
ment pouvoir en faire profiter ses proches, mais également nouer de nouvelles alliances, agrandir le cercle social des interdépendances.

Ainsi, un migrant enrichi et revenu dans le pays d'origine, pourra-t-il, par exemple financer le départ de nouveaux migrants, ce qui rendra ceux-là ainsi que leur famille redevables. De la même façon, les cadeaux qu'il apportera lors des différentes cérémonies festives, telles que les mariages et les circoncisions, ainsi que la magnificence avec laquelle lui-même organisera ces rassemblements, pousseront ses hôtes à l'inviter dans d'autres circonstances, et à renforcer les liens ; connaître un homme fortuné étant de bon aloi.

Ces relations sont principalement établies au regard de la position financière de l'ex-migrant, et non de son charisme, de sa loyauté, de sa sagesse ou surtout de son âge, qui sont pourtant des caractéristiques prépondérantes des figures de l'autorité en Ouzbékistan. Ici, il s'agit de connaître des hommes fortunés et de se faire reconnaître comme riche. Plus un homme est riche, plus il est amené à dépenser de l'argent pour les autres, dans un système de dépenses en escalade. Il faut donner toujours plus que l'autre, organiser des fêtes encore plus somptueuses, se montrer encore plus généreux dans les dons. Ceci n'est pas sans rappeler le potlatch décrit par Mauss (2001), ce système de dons et de contre-dons sur un mode antagoniste, les bienfaiteurs cherchant sans cesse à aller plus loin que l'autre dans l'offre.

Il est habituel d'entendre dire, en Ouzbékistan, que la richesse d'un homme se mesure davantage à son réseau social qu'à son portefeuille. Le paradoxe des Nouveaux Ouzbeks réside dans le fait que c'est justement leur argent qui leur a permis d'étendre et de consolider leur réseau social.

\section{Les célébrations rituelles pour éviter la chute}

Les migrants ont acquis, par l'argent ramené des pays d'accueil, une nouvelle façon de marquer leur espace social. Leur nouveau statut de " nouvel Ouzbek » est pourtant précaire : en cas de paupérisation, ces relations superficielles s'effaceront. C'est en tout cas ce que craignent les migrants. Dès lors, des protections symboliques sont recherchées pour éviter la chute, prévenir la déchéance.

J'ai pu assister à différents rites et les migrants m'en ont fait découvrir d'autres. Le plus commun est le is ou hatimkoran, c'est-à-dire littéralement « lecture du Coran ». Un animal, poulet, mouton ou bœuf, est sacrifié, le sang servant à marquer l'endroit qu'il convient de protéger. Un mollah récite une prière. La cérémonie se conclut par un repas, où l'animal sacrifié est cuisiné et partagé par les convives. Il convient pour les migrants de chercher une garantie symbolique de conserver leur statut acquis par la migration. Il s'agit surtout de se prémunir du mauvais œil. En effet, plus l'on est regardé par les autres, plus on est admiré, plus le risque est grand de perdre ce que l'on a, de tomber malade, de redescendre l'échelle sociale. Les migrants enrichis attirent des regards envieux, dont il faut se prémunir pour éviter la chute. 
Des réunions féminines sont organisées par les épouses des migrants ${ }^{9}$, dans ce même but, tels que les muchkulkuchod ou bibisechanbe. Ce sont des cérémonies religieuses menées par une bibihalfa, femme pieuse qui équivaut plus ou moins au mollah pour les femmes. Les participantes et surtout l'organisatrice espèrent être ainsi protégées des aléas de l'existence.

Tous ces éléments religieux doivent permettre d'éviter ce que les migrants craignent le plus : la chute, à la fois sociale et financière. Par ailleurs, les investissements de l'argent de la migration sont effectués dans l'espoir de se maintenir dans une situation économique confortable. Mais les exemples des migrants malheureux continuent à les inquiéter.

\section{Significations et enseignements des échecs migratoires}

Il convient de revenir ici sur les cas d'échecs au cours des migrations. Dans les rites de passage, les candidats ne passent pas tous l'épreuve avec réussite. Certains ne parviennent pas au terme de l'initiation, notamment quand le rite est particulièrement éprouvant pour le corps, et cet échec peut se traduire par le décès. En migration, il n'y a évidemment pas de mutilations ou autre marquage du corps ritualisé. Cependant, l'affaiblissement et l'amaigrissement peuvent être considérés comme des traces visibles du passage en migration. Les marques physiques (cicatrices, membres amputés, etc.) résultant d'agressions racistes en Russie en font aussi partie, comme me l'explique une étudiante tadjike de Samarkand rencontrée à Paris. Elle me demande ainsi de me rendre chez ses parents et d'essayer de faire parler son père pour connaître son histoire migratoire restée secrète :

"Tu sais, avec mon père, on ne sait pas vraiment ce qu'il s'est passé. Il est parti pour travailler en Russie. Et puis, on n'a pas eu de nouvelles. Ma mère était très inquiète, nous [les enfants du couple], on ne comprenait pas très bien ce qu'il se passait. On ne recevait pas du tout d'argent, mes grands-parents ont dû nous aider

à vivre. On a essayé de le retrouver, en interrogeant des gens qui étaient allés à

Moscou et auraient pu le rencontrer. On a même fait une émission de télé pour lui demander de revenir, on a pleuré, c'était dur. On a attendu, attendu. Finalement, on a pensé qu'il était mort, et on a réorganisé notre vie sans lui.

Un soir, il a frappé à la porte. Il est entré. Il lui manquait des doigts à la main. Il n'a jamais rien expliqué, on ne sait absolument rien. Ma mère a décidé de ne pas lui poser de questions. Moi, j'ai essayé, mais alors les larmes lui montent aux yeux et il ne répond pas. Son retour, ça a été une grande joie pour nous, de voir qu'il était bien vivant, mais après, il y a eu des problèmes. Surtout avec mon frère, qui a appris entre temps qu'il était en réalité le fils de ma tante, et que ma cousine est en fait ma sœur biologique. Du coup, il ne supporte plus l'autorité de mon père, car il s'est senti abandonné. C'est très dur pour mon père de ne pas être respecté par son propre fils... enfin, qu'il considère comme son fils. Surtout qu'en Ouzbékistan, tu le sais, c'est très important.

9 «En Asie centrale, les hommes et les femmes ne pratiquent pas de la même manière et ont ainsi un rapport au religieux différent. [...] Le nombre d'édifices religieux conçus pour les femmes et destinés à leur offrir une éducation religieuse est considérablement inférieur à celui conçu pour les hommes » (Fathi, $2004: 239-240$ ). 
Moi, je comprends qu'il a dû vivre des moments très difficiles, et qu'il ne veut pas nous les raconter. Mais toi, tu es étrangère, et tu travailles sur les migrations, peut-être qu'il pourra t'en parler et que tu me diras ce qu'il s'est vraiment passé? 》.

L'émission russe « Ždi menâ. Nacional'naâ služba vzaimogo poiska lûdej » ${ }^{10}$ [Attends moi. Service national de recherche mutuelle de personnes] est un moyen souvent utilisé par des familles centrasiatiques dans l'espoir de retrouver un parent parti travailler en Russie. Certaines familles restent ainsi sans recevoir aucune nouvelle, ou encore apprennent le décès de leur proche, sans plus de détails sur les circonstances de sa mort ${ }^{11}$. Cependant, ces problèmes liés à la migration ne sont pas évoqués clairement par les familles restées en Ouzbékistan, car à l'inquiétude due à ce silence s'ajoute la honte de ce qui est considéré comme un échec et, parfois, la peur de ne pouvoir rembourser les dettes contractées pour le départ.

Face à ce silence, la plupart des gens n'insistent pas et ne posent pas plus de questions. Mais mon passage en Ouzbékistan a été l'occasion pour certains de lever une partie du voile opaque qui empêche de savoir ce qui se passe « vraiment » à l'étranger. En effet, à la suite de mes trois terrains dans les pays d'accueil, je suis donc repartie en Ouzbékistan. Les questions concernant la migration pouvaient m'être adressées plus facilement en ma qualité d'hôte étrangère, donc non impliquée dans les mêmes logiques socioculturelles qu'un migrant ouzbek, mais ayant vu différentes situations migratoires de mes propres yeux. Répondre à ces interrogations générales ne me posait pas trop de problèmes, bien que cela rende le chercheur démiurge de son propre sujet. Cependant, l'engagement était bien différent quand les questions portaient sur un migrant spécifique. Comment répondre aux familles, ne sachant pas ce que le migrant avait lui-même dévoilé ou non de sa vie à l'étranger?

Le problème s'est en réalité posé de manière moins aiguë que je ne le craignais, les familles préférant souvent être rassurées sur l'état de santé et le moral du migrant, plus que sur ses conditions exactes de vie à l'étranger. Mais il m'est arrivé en Ouzbékistan que des familles fassent venir une connaissance, un ami ou un voisin, revenue de l'étranger, pour que je puisse l'interroger devant eux, et en savoir ainsi davantage sur son parcours.

En définitive, l'échec de la migration peut se traduire par une absence de nouvelles due soit au décès, soit à l'impossibilité d'en donner, soit à la honte du migrant d'être dans une situation matérielle peu enviable. Cela peut nous faire songer à ce que décrivait Van Gennep (1981 : 108) : "le novice est considéré comme mort, et il reste mort pendant la durée du noviciat. Celui-ci dure un temps plus ou moins long et consiste en un affaiblissement corporel et mental du novice. [...] [Ensuite,] on le ressuscite et on lui apprend à vivre, mais autrement que pendant l'enfance ». Les initiés qui s'en sortent sont ensuite agrégés à un nouveau groupe à leur retour dans le pays d'origine.

10 Cf. http://poisk.vid.ru/, consulté le 20/07/2008.

11 «Focus on southern labour migration », http://www.uzland.info/2005/march/09/02.htm, consulté le 20/07/2008. 


\section{INVESTISSEMENT DE L'ARGENT ET BOULEVERSEMENTS ÉCONOMIQUES}

L'argent des migrations joue un rôle crucial dans l'économie du pays d'origine. Mais comment est-il investi concrètement et quel apport représente-t-il pour le pays ? L'une des principales dépenses est le mariage des enfants du migrant ou des migrants eux-mêmes, fête particulièrement onéreuse en Ouzbékistan. Ensuite, il s'agit du logement, l'achat immobilier représentant un investissement durable pour la famille. Il peut aussi être injecté dans des secteurs lucratifs, dans des projets potentiellement rémunérateurs. Enfin, il peut servir à acquérir des preuves de richesse, afin de démontrer au voisinage combien la migration a été bénéfique pour l'amélioration de la vie quotidienne.

\section{Mariages et autres célébrations}

Le mariage est célébré avec faste en Ouzbékistan et les festivités qui y sont liées sont particulièrement onéreuses. De nombreux Ouzbeks s'endettent pour parvenir à faire face à de telles dépenses. L'argent économisé au cours de la migration permet d'accueillir comme il se doit les invités.

\section{Importance et intérêt de la dépense}

En Ouzbékistan, comme dans bien d'autres pays, les passages de la vie sociale considérés comme les plus déterminants sont caractérisés par des regroupements festifs. Ils sont destinés à marquer ces transitions et à les effectuer en respectant ce que l'on peut rapidement nommer les traditions, bien que ce terme ne doive pas être compris comme relevant d'une histoire figée, mais au contraire comme une adaptation permanente entre les valeurs culturelles et les possibilités contemporaines de leur réalisation.

Plusieurs événements sont ainsi célébrés avec faste, différemment selon le sexe des individus. Ruffier (2002 : 52, 79) a analysé ces fêtes, qu'il qualifie dans le titre de son ouvrage d' " espaces de construction des identités et des solidarités » : "les Ouzbeks donnent comme prétexte à l'organisation d'une to'y l'accouchement du premier enfant tug'ich to'yi, la mise au berceau - beshik to'yi, la circoncision - sunnat to'yi, le mariage - nikoh to'yi, l'installation dans une nouvelle maison - hovli to'yi, un anniversaire important (cinquante ans, soixante-trois ans [âge de la mort du Prophète], soixante-dix ans) - jubile to'yi. [...] Les quatre étapes [principales] du cycle des to'y, circoncision/ mariage/circoncision/mariage, dans la vie d'un individu tout d'abord sujet de la célébration, puis organisateur, forment clairement un cycle d'insertion progressive dans une communauté de vécu ». On voit ici que cela est envisagé du point de vue masculin, avec la circoncision.

Le point d'orgue de la vie sociale d'un individu est sans doute son mariage, qui doit le faire définitivement passer au statut de père ou de mère de famille, et celui qu'il organisera pour ses enfants. La fête se déroule traditionnellement chez les parents de l'époux. Ils reçoivent les invités dans la cour de leur maison ou à l'extérieur de celle-ci ou encore, pour certains, dans un restaurant. Cette dernière possibilité est très prisée par 
les « nouveaux Ouzbeks » car il s'agit alors d'un mariage encore plus onéreux et visible par tous. Il y a différentes étapes dans le mariage et l'enregistrement administratif, appelé ZAGS, n'est que le prélude à une longue festivité. Bien que la famille du marié prenne en charge la réception principale, celle de la mariée est aussi fortement impliquée, par un jeu de dons et de contre-dons entre les deux parties.

Les sommes engagées dans la réception des invités d'un mariage sont très importantes et plusieurs Ouzbeks m'ont affirmé qu'un seul mariage représentait parfois jusqu'à une dizaine d'années d'économie pour un père. Ce sacrifice est consenti par de très nombreux parents, qui sortent parfois ruinés de la noce de leurs enfants, mais fiers. Aujourd'hui, certains migrants partent donc pour pouvoir réunir plus rapidement cette somme et ainsi marier dignement leurs enfants. Il s'agit d'une question d'honneur, et non, au sens propre, d'une tentative de lutte pour sa survie. À moins que l'on puisse considérer l'organisation d'un mariage comme une nécessité pour la survie sociale d'un parent, ce qui se rapproche assez bien de ce que j'ai pu observer. Jadis, il était d'usage que ce soit les parents qui assument seuls les dépenses liées au mariage. Actuellement, certains jeunes gens décident de soulager leurs parents et de partir à l'étranger pour réunir eux-mêmes la somme nécessaire à l'organisation d'un mariage « digne de ce nom ». Mais qu'est-ce qui se trame dans ces réunions festives?

\section{Un tissage de réseaux sociaux de plus en plus onéreux}

Ruffier qualifie les fêtes ouzbèkes, d'« espaces de construction des identités et des solidarités ». Cela représente bien l'enjeu principal d'un mariage. Au-delà de la construction d'un foyer pour les époux, qui fondent leur propre famille, le mariage en lui-même est la vitrine de la situation sociale des parents, de leur capacité à réunir des gens et à les recevoir avec faste. Le capital financier est prépondérant, puisque les sommes engagées sont considérables. Le repas est pantagruélique : des animaux sont généralement abattus pour confectionner les plats principaux, dont l'incontournable plov. En outre, les boissons, alcoolisées et non-alcoolisées, sont servies à foison, un orchestre anime la plupart du temps les festivités, des danseuses professionnelles sont embauchées, et le tout est souvent filmé par un caméraman engagé pour l'occasion.

Les invités ne se comptent pas. Dans les villages, des crieurs annoncent la date des réjouissances et tous les habitants qui connaissent un tant soit peu les protagonistes se déplaceront pour l'événement. Souvent, les invités, si on peut les nommer ainsi, quittent la table une fois leur repas avalé afin de céder leur place aux nouveaux arrivants, dans un mouvement incessant d'arrivées de nouveaux convives. Des tentatives de régulation des mariages ont été effectuées par le gouvernement, qui entendait limiter le nombre de convives, la quantité d'alcool proposée ainsi que le montant général des dépenses. Mais les lois sont une fois de plus contournées. Les responsables du comité des mahalla, chargés de faire respecter ces restrictions, s'avèrent le plus souvent complaisants et les mariages continuent à représenter une fête grandiose.

Pétric (2006 : 175) évoque l'existence de cahiers de comptes, appelés sharbatnoma, dans lesquels sont inscrits les cadeaux donnés et reçus. Ils permettent aux familles de garder trace des échanges, et de savoir ce qu'il convient de " rendre » à untel lors 
d'une prochaine festivité. Je fais ici référence au cycle du don (donner, recevoir, rendre), mis à jour par Mauss (2001 : 143-279). Les invités offrent divers présents aux jeunes mariés : tapis, matériel électroménager, argent, etc. En retour, ces derniers ou leurs parents donneront dans une prochaine fête un cadeau de valeur semblable ou supérieure.

Or ces cadeaux, chez les migrants, sont de plus en plus importants et de plus en plus chers. C'est ce que me confie Muhabat, alors que nous assistons ensemble au mariage d'une jeune fille de sa parenté. J'avais rencontré sa belle-sœur à Séoul et son frère à Samarkand. Le mariage fut pour moi l'occasion de faire connaissance avec plusieurs membres de cette famille qui bénéficie des retombées financières de la migration :

"Tu ne connais peut-être pas mes trois autres frères qui vont régulièrement à Séoul pour travailler? Ensemble, ils font du business de voiture, qu'ils envoient en Russie ou en Chine. Ils ont le visa du business, le D8. Deux de mes belles-sœurs [épouses de ses frères] sont aussi allées là-bas pour travailler. Moi aussi,j'aimerais partir là-bas avec mon mari. On a eu cinq filles, et les deux dernières sont des jumelles. Je voudrais m'arrêter là, mais mon mari veut un fils. Il va falloir qu'on pense à leur mariage, cela coûte cher. Et puis, cela nous coûte aussi très cher d'assister aux mariages des autres : on ne peut quand même pas venir les mains vides!

Ici, les salaires ne suffisent pas.

Tiens, regarde, c'est eux les parents de la mariée [Muhabat me montre du doigt la photo d'un couple qui apparaît sur un écran géant dans la salle de restaurant où nous nous trouvons]. Ça fait dix ans qu'ils sont partis en Corée, et qu'ils n'ont pas remis les pieds en Ouzbékistan. Depuis ses huit ans, la mariée [qui a dix-huit ans] vit avec ses grands-parents. C'est eux qui ont organisé le mariage pour elle. C'est un beau mariage, c'est sûr, mais quand même, les parents devraient être là ! Ce n'est pas dans nos traditions de se marier sans ses parents!».

Ainsi, une des causes de la migration peut être le besoin non seulement d'organiser pour ses enfants une fête digne, mais aussi de rendre les honneurs lorsque l'on est invité à une cérémonie. Ici, Muhabat est prise dans les réseaux de ses frères, tous migrants. Les cadeaux sont très onéreux, et Muhabat et son mari peinent à suivre le rythme effréné des dons. Enrichis, les migrants organisent des fêtes-vitrines, destinées à rendre visible leur nouveau statut financier. Ici, le cas est particulièrement intéressant, car les parents ont délégué à leurs propres parents l'organisation des festivités, qu'ils financent, mais auxquelles ils ne peuvent pas assister. Étant illégaux en Corée du Sud, mais y exerçant une activité lucrative, ils souhaitent y rester plus longtemps sans pouvoir revenir en Ouzbékistan, de crainte de se voir interdire de séjourner à l'avenir sur le territoire coréen.

\section{Logement et amélioration du cadre de vie}

Pour beaucoup de migrants, l'achat d'un logement représente un investissement important de l'argent économisé en migration. Il s'agit d'abord de s'assurer de rester dans un lieu qui leur appartient, d'offrir un toit à leur famille, mais aussi de s'ancrer dans un territoire spécifique. Enfin, il permet aussi aux migrants de donner à voir leur nouveau statut. 


\section{L'ancrage dans un territoire (ville et mahalla)}

Ainsi, pour certains migrants nés dans un village, posséder un logement dans la ville est un gage de non-retour au village. De nombreux qishloqi venus travailler dans une ville, comme j'ai pu le voir à Samarkand, rêvent de s'acheter un " appartement russe ", c'est-à-dire un appartement dans une barre d'immeubles. Il s'agit ici d'une représentation du confort, du luxe à l'occidentale avec «toutes les commodités », comme aiment à le souligner les néo-citadins : gaz, électricité, eau courante et même eau chaude, sanitaire relié au tout-à-l'égout. Se pose toutefois la question de l'autorisation, la propiska à obtenir avant de pouvoir s'installer ${ }^{12}$.

$\mathrm{Au}$ contraire, certains migrants reviennent de l'étranger en souhaitant s'acheter une « maison sur cour », parfois appelée aussi « maison ouzbèke », mais en périphérie de la ville, pas à la campagne. Il s'agit pour eux de revenir aux maisons " traditionnelles ", comme plusieurs d'entre eux me l'ont expliqué, mais en y apportant les commodités des « appartements russes ». La maison sur cour permet d'y organiser des festivités, et d'accueillir la famille élargie, ce qui n'est pas à proprement parler impossible dans un « appartement russe », mais moins confortable car plus exigu.

Le summum du luxe est représenté par les " maisons américaines ». Il s'agit de constructions modernes, souvent bâties sur mesure, imaginées par des architectes qui puisent dans les représentations du luxe à l'ouzbek. Frises, dorures, imitation de marbre, moulages en stuc, bois sculptés sont autant d'ornements indispensables. L'essentiel est de montrer la richesse des matériaux employés et d'y ajouter une touche occidentale : monumentale table en bois, escalier au milieu de la pièce, tonnelle en fer forgé, autant d'éléments de décoration souvent inutilisables ou peu fonctionnels, mais estimés par leurs propriétaires. J'ai ainsi été invitée à visiter une demeure de Namangan, construite grâce à l'argent envoyé par un migrant à Séoul. C'est Alikul qui me guide, fier de me faire découvrir la demeure de sa grand-mère :

"Voilà ma grand-mère, mon oncle, sa femme et leurs enfants [nous sommes dans une bâtisse à l'entrée de la propriété. Elle comporte deux pièces. C'est le seul bâtiment éclairé à notre arrivée]. Vous allez voir maintenant la maison, comme elle est immense. C'est une maison américaine, comme dans les feuilletons. [La visite se poursuit, avec toute la famille. Ils ne savent pas toujours où se trouvent les interrupteurs permettant d'éclairer les différentes pièces que nous visitons. Ils attendent visiblement ma réaction devant le majestueux escalier en colimaçon qui trône au milieu du séjour et permet d'accéder à la mezzanine.] Voilà, c'est notre maison

coréenne!".

12 Article de Roberta Cohen, «Freedom of movement », United States Mission to the OSCE (US Delegation to the Human Dimension Implementation Meeting), 07/10/2003: «In Uzbekistan, the government [...] severely limits the right to change permanent residence in country. Permission from local authorities is required in order to resettle in a new city, and the authorities rarely grant permission to those who wish to move to Tashkent. Local observers report that a bribe of roughly $\$ 100$ (96,529 soum) is necessary to obtain the required registration documents. Again, this is contrary to Uzbekistan's Vienna commitment that everyone has the right to freedom of movement and residence within the borders of each State ", http://osce.usmission.gov/archive/2003/10/ FREEDOM_OF_MOVEMENT.pdf, consulté le 24/07/2008. 
La maison américaine est devenue, en fin de visite, une maison coréenne. En réalité, j'ai entendu plusieurs fois la première expression, plus rarement la seconde. Il s'agit de désigner un logement directement inspiré des lieux de tournage de feuilletons américains, comme me l'ont affirmé plusieurs migrants. En réalité, il s'agit moins d'un habitat que d'un décor. Ici, personne ne dormait dans la maison principale, la «maison américaine » : la grand-mère, son fils, sa bru et ses quatre petits-enfants vivent dans la petite bâtisse de l'entrée. Il s'agit de paraître, la maison s'avérant fonctionner comme une vitrine de la réussite du migrant.

L'importance prise par l'escalier m'a beaucoup surprise, tant son aspect imposant me paraissait incongru, car il occupait le milieu d'un salon, comme s'il en constituait l'attrait principal. Je pense qu'en fait, il sert particulièrement à y prendre des photos, et représente en lui-même ce que mes interlocuteurs estiment être le design américain. J'ai compris cela en me rendant avec Dilnoza chez un photographe de Samarkand. Elle souhaitait faire parvenir à son époux, qui travaille à Séoul, son portrait. Avant d'être photographiée par le professionnel, elle devait choisir le décor qui servirait de toile de fond à son portrait. Le choix était large : gratte-ciels américains, Tour Eiffel, cottage anglais, palmiers, jardins européens, paysages de montagne, vue d'Hollywood. Dilnoza choisit finalement deux fonds : l'un représente une luxueuse voiture de sport garée devant une maison, l'autre est un cliché où figure l'intérieur d'une maison, le tout faisant, comme le dit Dilnoza elle-même, " très américain ». Dilnoza a emmené des vêtements pour pouvoir se changer. Elle désirait être prise en photo dans un ensemble veste et pantalon blancs, et a donc laissé sa robe de tissu ouzbek de côté le temps que le photographe l'immortalise.

Une hiérarchisation officieuse des logements serait la suivante : maison ouzbèke dans la campagne, appartement russe dans la ville, maison américaine (ou coréenne) dans la ville, maison ouzbèke dans la ville. Cette échelle renvoie à la hiérarchisation des pays d'accueil : la Russie, puis la Corée, puis les États-Unis, destinations du moins au plus facile d'accès et du moins au plus lucratives. Plus loin les migrants partent, plus importante sera la somme rapportée. Et parallèlement, plus ils émigrent loin, plus les logements achetés en Ouzbékistan seront prestigieux et éloignés de la campagne. La maison américaine semble donc être une demeure vitrine, symbole du changement de statut.

\section{Des logements prestigieux qui restent vides}

Au cours de mes terrains dans les trois destinations, il m'est très souvent arrivée d'être invitée par mes interlocuteurs à rendre visite à leur famille en Ouzbékistan. Mais j'ai été très surprise par le nombre de migrants qui me proposaient de disposer d'un logement resté vacant en leur absence. Ce fut le cas avec Robiâ :

Robiâ : "Si vous voulez, vous pouvez téléphoner à mes parents quand vous serez à Samarkand. J'expliquerai à ma mère que l'on s'est rencontré ici et elle sera ravie d'accueillir un mehmon [o. : invité] de France! Elle vit avec mes enfants, vous les verrez comme ça! Ils me manquent tellement. "

S. : "Ils habitent où, dans Samarkand?»

Robiâ : "On leur a acheté une maison au Registan [sous-entendu : avec l'argent gagné en migration par elle et son mari]. Mais on a aussi un appartement rue Gagarine, près de la gare. Vous pourrez loger là si vous voulez, il n'y a personne qui l'occupe pour l'instant ».

REMI 2010 (26) 3 pp. 59-84 
Deux logements ont donc été achetés grâce à l'argent de la migration. Un seul est utilisé par la famille. L'autre, l'appartement, reste vide, en attendant que les migrants regagnent le pays d'origine. Le discours est identique de la part de Samira :

"Mes parents sont à Samarkand, mais je vois ma mère environ une fois par mois, car elle vient ici pour faire du business. Moi-même je reviens à peu près deux ou trois fois par an en Ouzbékistan. On a trois maisons à Samarkand, mais elles sont vides, car mes parents préfèrent vivre dans leur maison. Donc si vous allez à

Samarkand, dites-le-nous ».

Comme le rappelle G. Pincent (2008), « depuis l'indépendance de l'Ouzbékistan en 1991, la notion de "propriété privée" a pris un sens nouveau. Certes, le sol appartient à l'État. Mais les murs des habitations sont détenus par la population. Leurs propriétaires considèrent qu'ils peuvent en faire ce qu'ils veulent. On assiste donc depuis 1991 à une explosion des chantiers dans les villes centrasiatiques [...]. Il existe [...] un système de contrôle des constructions, reposant sur le permis de construire. Mais beaucoup contournent ces lois. L'accès à la propriété privée, même limité par un labyrinthe administratif, a ainsi encouragé les transformations totales ou partielles opérées dans les maisons traditionnelles. Il faut les réparer, les agrandir, améliorer leur confort ou montrer la richesse familiale ».

Cette course à l'amélioration de la demeure semble avoir davantage pour but de posséder un bien visible par tous et prestigieux que de l'habiter réellement. Il peut s'agir d'un investissement pour le futur, comme Tursun :

«Je me suis fait construire une maison à Kokand, pour plus tard, quand je serai marié. Pour l'instant, elle est vide. Quand je rentrerai, je préférerais vivre chez mes parents d'abord, et après, on verra ».

Ces maisons sans habitants ne doivent pas pourtant apparaître comme inutiles, puisqu'elles servent à s'attirer une certaine reconnaissance sociale.

\section{Vers un nouveau mode d'habitat?}

Vivre dans un nouveau logement, que l'on vient d'acquérir, équivaut aussi bien souvent à changer ses habitudes domestiques. Tout d'abord, il peut s'agir de changer de lieu de vie, d'habiter désormais dans une ville différente de celle où le migrant séjournait avant le départ. Mais surtout, la forme du logement et son aménagement peuvent différer. Ainsi, habiter un appartement russe en centre-ville de Tachkent signifie-t-il changer significativement de mode d'habitat pour celui qui est né dans une maison en terre d'un qishloq.

L'aménagement est censé montrer, une fois de plus la richesse des propriétaires. Bekzod s'est ainsi acheté un appartement pour lui, à Tachkent, et a fait construire chez ses parents, à Qarchi, un sauna à l'extérieur de la maison. Quand je me suis rendue chez eux, j'ai pu constater qu'en effet, une magnifique pièce en bois tout à fait fonctionnelle, semble-t-il, se dressait dans le jardin. Les parents de Bekzod me l'ont fait visiter, mais il m’a semblé que le sauna n'était pas très souvent utilisé. De la même façon, voici ce que m'a confié Nodira, qui habite au cinquième étage d'un grand immeuble soviétique dans 
Tachkent et me fait visiter son appartement :

"Vous voyez, il y a dix pièces. Depuis deux ans, j'ai fait beaucoup de travaux dans cet appartement, sans le dire à Ališer. Il aura la surprise quand il rentrera [de New York]. Je ne m'attendais pas à ce que ce soit aussi dur de diriger des travaux, quand on est une femme! Mais je ne me plains pas, j'ai bientôt terminé ce que je voulais faire. Seulement, je voulais installer un jacuzzi et il y a deux problèmes. Déjà, ça ne passait pas dans l'ascenseur, et dix hommes auraient été nécessaires pour le monter par l'escalier. Et puis de toute façon, ça risquait de faire sauter l'électricité à chaque fois qu'on l'allume. C'est une machine qui consomme beaucoup d'énergie. Donc j'ai préféré finalement faire installer une baignoire à trois angles. C'est aussi bien comme ça. Mais qu'est-ce que c'est fatigant de s'occuper de tout ça, et de voir l'appartement sens dessus dessous pendant les travaux! ».

Cet exemple est assez étonnant, en ce qu'il nous montre une femme qui décide seule de l'utilisation de l'argent envoyé par son époux qui travaille à New York. Elle prend en charge les travaux et la transformation de l'appartement en un luxueux logement, sans en avertir son époux.

Enfin, des installations informatiques sont prévues dans plusieurs logements de migrants. Ainsi, Ziëd s'est-il acheté à Séoul un ordinateur et a-t-il fait installer une connexion Internet à domicile pour que lui, ses enfants et le reste de la famille puissent en profiter. De même, Tahir a fait acheter à ses parents, depuis New York, un ordinateur fixe et un ordinateur portable. L'aménagement des logements consiste donc à faire voir des objets de valeurs, pour démontrer aux visiteurs l'importance de la richesse de ses hôtes. Les voitures jouent aussi ce rôle.

Parfois, ce sont aussi des signes qui rappellent le pays d'accueil qui se trouvent exposés par les familles. Les photos touristiques, par exemple, sont parfois mises sous cadre et montrées. Dans la famille de Bekzod, la présence d'un parent aux États-Unis est particulièrement valorisée, et un autocollant aux couleurs de Columbia University a été placé à l'arrière de la voiture. Dans la famille de Tursun, l'appareil servant à cuire le riz, ramené de Corée, est souvent montré aux invités, comme une curiosité. Dès lors, il semble que le logement puisse être envisagé comme une vitrine de réussite matérielle par le passage à l'étranger.

\section{CONCLUSION}

Devenu « nouvel Ouzbek », l'ex-migrant n'informe personne de ce qui s'est réellement déroulé à l'étranger. Il rentre enrichi, et la manière dont il a perçu cet argent n'est jamais éclairée. La zone d'ombre de l'entre-deux, de la période liminaire qu'est la migration, est laissée de côté, au profit de l'insistance sur les marques du nouveau statut social des ex-migrants, reconnus comme sortis victorieux de leur initiation secrète.

Les migrants de retour dans leur pays d'origine semblent avoir acquis une grande visibilité et du fait de leur richesse sont investis d'une mission particulière pour s'associer 
à toutes sortes de projets. Ce dynamisme pourrait en quelque sorte être considéré comme une contrepartie à l'abandon de la patrie : partis loin de chez eux pendant de longues années, ils en reviennent plus attachés encore à leur ouzbékité, et déterminés à jouer un rôle prépondérant dans l'Ouzbékistan post-soviétique. Ainsi, les rapports de pouvoir sont redéfinis au sein de la famille. Les migrants ne peuvent retrouver la même position de père, d'époux et de fils qu'ils occupaient avant leur départ, alors que la maison a fonctionné sans eux pendant parfois de nombreuses années. Ils changent de statut au niveau du mahalla car, devenus « nouveaux Ouzbeks », c'est-à-dire enrichis, ils tissent un réseau d'interdépendance qui les place en position de force. Sollicités pour leur argent, leur capital social s'accroît considérablement.

D’un autre côté, ceux qui n'ont pas amassé un capital suffisant pour partir, s'enfoncent dans le néant, et perdent leur identité sociale. Quant aux familles des migrants, elles suivent une trajectoire identitaire tout à fait surprenante, en acquérant toutes les apparences de l'occidentalisation, rigoureusement à l'inverse des migrants de retour.

À partir de ce paradoxe se dégage la conclusion suivante : en accomplissant le rituel migratoire, les migrants se constituent une identité de transition : ils bouleversent les rapports sociaux et la hiérarchie domestique, et pourtant deviennent les garants d'une ouzbékité imaginée; ils vont à l'étranger, mais sont indifférents aux valeurs des pays où ils ont vécu et permettent, grâce aux capitaux rapportés, de procéder aux rites qui marquent le plus fortement l'ouzbékité (mariage, réseau de dons et contre-dons, etc.). Au contraire, ceux qui sont restés ne se situent pas dans cette identité de transition, mais continuent d'imaginer un Occident dont il faudrait adopter les valeurs, au détriment de sa propre identité.

\section{Références bibliographiques}

ADELKHAH Fariba et OLSZEWSKA Zuzanna (2006) Les Afghans iraniens, Études du CERI, 125, $42 \mathrm{p}$.

ALTHANS Birgit, AUDHEIM Kathrin, WULF Christoph, et al. (2004) Penser les pratiques sociales comme rituels : ethnographie et genèse de communautés, Paris, L'Harmattan.

AUGÉ Marc et Daniel FABRE (1987) D'un rite à l'autre. Entretien entre Marc Augé et Daniel Fabre, Terrain, 8, dossier « Rituels contemporains », pp. 71-76.

BELL Catherine (1992) Ritual theory, ritual practice, New York, Oxford, Oxford University Press.

BELMONT Nicole (1986) La notion de rite de passage, in Pierre Centlivres et Jacques Hainard Dir., Les rites de passage aujourd'hui. Actes du colloque de Neuchâtel 1981, Lausanne, L'Age d'homme, pp. 9-17.

BENNEGADI Rachid et BOURDILLON François (1990) La santé des travailleurs migrants en France : aspects médico-sociaux et anthropologiques, Revue Européenne des migrations internationales, 6 (3), pp. 129-144.

BOURDIEU Pierre (1982) Les rites comme actes d'institution, Actes de la Recherche en Sciences Sociales, 43 (1), pp. 58-63.

CENTLIVRES Pierre (2000) Portée et limites de la notion de diaspora, Cahiers d'Études sur la Méditerranée et le Monde Turco-Iranien, 30, pp. 5-12. 
FATHI Habiba (2004) Femmes d'autorité dans l'Asie centrale contemporaine. Quête des ancêtres et recompositions identitaires dans l'islam postsoviétique, Maisonneuve \& Larose, IFEAC, Paris, $348 \mathrm{p}$.

GLUCKMAN Max (1962) Les rites de passage, in Max Gluckman Dir., Essays on the ritual of social relations, Manchester, Manchester University Press.

KAUFMANN Jean-Claude (2006) L'entretien compréhensif, Paris, Armand Colin.

LARUELLE Marlène (2006) Le nouveau rôle de la Russie en Asie centrale : les migrations de travail des Centre-asiatiques vers la Fédération russe, La Revue internationale et stratégique, 64 (4), pp. 133-141.

MASSOT Sophie (2009) Enjeux politiques et identitaires dans la migration internationale des Ouzbeks vers trois pôles urbains (Moscou, Séoul, New York), thèse de doctorat ès Sciences Politiques, IEP/FNSP-CERI, Paris, sous la direction de Dominique Colas.

MAUSS Marcel (2007) Essai sur le don. Forme et raison de l'échange dans les sociétés archaïques, Réédition du texte paru en 1923-1924 dans l'Année sociologique, Quadrige, Presses Universitaires de France, Paris, introduction par Florence Weber.

PÉTRIC Boris-Mathieu (2002) Pouvoir, don et réseaux en Ouzbékistan post-soviétique, Paris, Presses Universitaires de France-Le Monde (Partage du savoir), 298 p.

PINCENT Guillemette, La réhabilitation fonctionnelle des villes pré-coloniales d'Ouzbékistan : un outil de domination politique ?, http://www.cybergeo.eu/index1750.html, consulté le $24 / 07 / 2008$.

POUJOL Catherine (2005) Ouzbékistan : la croisée des chemins, Paris, Belin, La Documentation française (Asie plurielle).

POUJOL Catherine (2006) Santé mentale et société en Asie centrale post-soviétique ou comment tenter de diagnostiquer un mal-être social ?, in Outre-Terre (dossier Asie antérieure), 16 (3), pp. 367-380.

REGAMEY Amandine (1999) A. Loukachenka au miroir des histoires drôles. http://www.cerisciencespo.com/archive/septem99/artar.pdf.

RUFFIER Arnaud (2007) Samarcand. Identités et espaces festifs en Ouzbékistan, Paris, Aux lieux d'être (Documents).

RUFFIER Arnaud (2002) To'y, gap, ziyofat et bayram, espaces de construction des identités et des solidarités en Ouzbékistan, thèse à l'école doctorale Asie mineure et intérieure, EHESS, sous la direction de Gilles Veinstein.

SEGALEN Martine (2005) Rites et rituels contemporains, Paris, Armand Colin (128).

TEMINE Émile (1999) France, terre d'immigration, Paris, Gallimard (Découvertes Gallimard/ Histoire).

VAN GENNEP Arnold (1981) Les rites de passage. Étude systématique des rites, Paris, A.-J. Picard.

WINKIN Yves (2005) La notion de rituel chez Goffman. De la cérémonie à la séquence, Hermès, 43, dossier Rituels, pp. 69-78.

WULF Christoph (2005) Introduction. Rituels. Performativité et dynamique des pratiques sociales, Hermès, 43, dossier Rituels, pp. 9-22.

WRIGHT Richard (1994) Rite of passage, New York, HarperCollins Publishers. 


\title{
Le retour des migrants ou l'émergence des «nouveaux Ouzbeks » : les effets d'un rite de transition
}

\author{
Sophie MASSOT
}

Les migrations économiques contemporaines des Ouzbeks vers Séoul, Moscou et New York mettent en jeu des réseaux socio-familiaux, qui placent le migrant, avant, pendant et après sa migration au cœur d'un rite de transition qui permet de faire émerger, lors du retour, la catégorie socio-économique des « nouveaux Ouzbeks ». L'agrégation du migrant à un groupe est l'enjeu crucial lors du retour, via l'initiation qu'a constituée la migration et dont il est sorti victorieux. L'article explore les changements de fonction et d'attitude des différents membres de la parenté qui conduisent le migrant à donner un sens à son nouveau statut social. L'auteure s'intéresse aussi aux placements de l'argent amassé pendant la migration. Deux postes de dépenses importants apparaissent : l'organisation de célébrations fastueuses et l'acquisition de logements prestigieux, qui servent à être vu et reconnu en tant que personne riche, en tant que « nouvel Ouzbek ». Enfin, l'argent des migrations peut être utilisé pour favoriser de nouvelles mobilités, celles notamment de personnes de l'entourage des ex-migrants. Le rite de transition génère alors de nouveaux rites, malgré le silence du migrant sur ce qui s'est réellement déroulé à l'étranger.

\section{Return of the Migrants or Apparition of the "New Uzbeks": The Effects of a Transition Ritual}

\author{
Sophie MASSOT
}

The contemporary economic migrations of the Uzbeks to Seoul, Moscow and New York bring into play social and family networks, which place migrants, before, during and after their migrations, in the heart of a rite of transition enabling the emergency, on their return, of a socioeconomic category referred to as "the new Uzbeks". Joining a group is the crucial stake for the migrants on their return via the initiation that such a migration constituted and which he came out of victoriously. First of all, the changes in the duties and behaviors of the various members in their families lead the migrants to give sense to his new social status. Then, they ought to invest the money accumulated during the migration. Two important items of spending emerge then: the organization of sumptuous celebrations and acquiring prestigious accommodation whose aim is to "show off" and be considered as a wealthy person, as a "new Uzbek". Lastly, the money issued from these migrations may set off a renewal of mobility since it is often used for the departure of members of the ex-migrants' family circle: the rite of transition generates then new rites, even though the migrants keep silent on what really happened when they were abroad. 


\section{El retorno de migrantes o la emergencia de los "nuevos Uzbecos": los efectos de un rito de transición}

\section{Sophie MASSOT}

Las migraciones económicas contemporáneas de los Uzbekos hacia Seúl, Moscú y Nueva York construyen redes sociales y familiares que ponen el migrante, antes, durante y después su migración al centro de un rito de transición que permite hacer hincapié, cuando el migrante vuelve, en la categoría socio-económica de los «nuevos Uzbekos». El punto fundamental de la vuelta es la admisión del migrante a un grupo, tras la iniciación que constituye la migración que logró hacer. Primero, los cambios de funciones y actitudes de los varios miembros de la familia permiten al migrante encontrar el sentido de su nuevo estatuto social. Secundo, el migrante suele invertir el dinero ahorrado durante la migración. Dos categorías de gastos importantes aparecen: la organización de fiestas tremendas y la compra de alojamientos prestigiosos. La meta es que la gente ven y reconocen el migrante como une persona rica, es decir un «nuevo Uzbeko». Al fin y al cabo, el dinero de las migraciones permite una nueva movilidad, puesto que está utilizado para la migración de las personas que rodean a los ex-migrantes: el rito de transición es la base de nuevos ritos, aunque el migrante suela quedar mudo en cuanto a lo que realmente ocurrió al extranjero. 